\title{
Broadband Modified Proximity Coupled Patch Antenna with Cavity-Backed Configuration
}

\author{
Deok Kyu Kong ${ }^{1} \cdot J^{2}$ aesik Kim ${ }^{1} \cdot$ Daewoong Woo $^{1} \cdot$ Young Joong Yoon ${ }^{2, *}$
}

\begin{abstract}
A modified proximity-coupled microstrip patch antenna with broad impedance bandwidth is proposed by incorporating proximitycoupled patch antenna into the rectangular open-ended microstrip feed line on a cavity structure. First we design a proximity-coupled microstrip antenna to have a wide bandwidth in the lower band centered at $7 \mathrm{GHz}$ using a cavity-backed ground. To broaden the bandwidth of the antenna to the upper band, we then apply a rectangular open-ended microstrip feed line, adjusting the relative position to the cavity to generate an additional resonance close to $10 \mathrm{GHz}$. The combination of lower and upper band design results in a broadband antenna with dimensions of $30 \mathrm{~mm} \times 30 \mathrm{~mm} \times 9 \mathrm{~mm}\left(0.9 \lambda_{0} \times 0.9 \lambda_{0} \times 0.27 \lambda_{0}\right)$ is designed where $\lambda_{0}$ corresponds to the free space wavelength at a center frequency of $9 \mathrm{GHz}$. The measurement results verify the broad impedance bandwidth (VSWR $\leq 2)$ of the antenna at $77 \%(5.6-12.6 \mathrm{GHz})$ while the broadside gain is maintained between $6 \mathrm{dBi}$ and $8 \mathrm{dBi}$ within the operational broad bandwidth.
\end{abstract}

Key Words: Broadband Antenna, Cavity-Backed Antenna, Microstrip Antenna, Proximity-Coupled Antenna.

\section{INTRODUCTION}

Microstrip patch antennas have been widely employed in wireless communication systems, mainly owing to their low cost, low profile, and easy fabrication. However, patch antennas usually have the disadvantage of a narrow bandwidth.

A proximity-coupled microstrip patch antenna has broadband characteristics compared to the probe-fed and edge-fed coupling methods. In the proximity-coupled microstrip configuration, the bandwidth can be increased by $10 \%-26 \%$ using a stacked radiator, L-probe feeding, and slot patch using stubs [14].

Placing the cavity-backed ground under the patch improves the bandwidth by reducing the surface wave and lowering the equivalent dielectric constant. Many microstrip antennas have been designed and studied on cavity structures to increase bandwidth [5-12]. In [6], a stacked patch with a cavity-backed ground plane enhanced the impedance matching and improved the bandwidth to $26 \%$. In [11], a narrow cavity was used to create an efficient coupling of patches with thick substrates, and the bandwidth was increased to $40 \%$ by increasing the coupling amount.

In [12], a cavity-backed patch antenna with proximity coupling fed using a conventional microstrip feed line is presented that achieves $69.6 \%$ voltage standing wave ratio (VSWR) bandwidth. There are three substrate layers and its height corresponds to $0.38 \lambda_{0}$, where $\lambda_{0}$ denotes the wavelength of the operational center frequency.

In this paper, we propose a broadband cavity-backed proximity-coupled patch antenna on two substrate layers modified

\footnotetext{
Manuscript received February 28, 2020 ; Revised July 5, 2020 ; Accepted August 31, 2020. (ID No. 20200228-024J)

${ }^{1}$ The 1 st R\&D Institute, Agency for Defense Development, Daejeon, Korea.

${ }^{2}$ Electrical and Electronic Engineering Department, Yonsei University, Seoul, Korea.

"Corresponding Author: Young Joong Yoon (e-mail: yjyoon@yonsei.ac.kr)
}

This is an Open-Access article distributed under the terms of the Creative Commons Attribution Non-Commercial License (http://creativecommons.org/licenses/by-nc/4.0) which permits unrestricted non-commercial use, distribution, and reproduction in any medium, provided the original work is properly cited.

(c) Copyright The Korean Institute of Electromagnetic Engineering and Science. All Rights Reserved. 
with a rectangular open-ended microstrip feed line (OEMF). The rectangular OEMF is defined as the combination of a rectangular patch and microstrip feed line. The rectangular OEMF technique can maintain the bandwidth and generate additional resonances to create a bandwidth expansion effect compared to those of conventional microstrip feed line. The proximitycoupled patch and rectangular OEMF are placed on upper and lower substrates on a cavity structure to generate multiple resonances in different frequency bands. The rectangular OEMF on the lower layer is designed to achieve an upper band VSWR bandwidth through the coupling with the cavity. The upper layer patch fed by the rectangular OEMF with the cavity realizes the lower band VSWR bandwidth. As a result, the proposed antenna realizes $77 \%$ VSWR bandwidth from $5.6 \mathrm{GHz}$ to 12.6 $\mathrm{GHz}$ with the dimensions of $0.9 \lambda_{0} \times 0.9 \lambda_{0} \times 0.27 \lambda_{0}$ (width $\times$ length $\times$ height), where $\lambda_{0}$ corresponds to the free space wavelength of $9 \mathrm{GHz}$.

\section{ANTENNA DESIGN}

The geometry and parameters of the antenna are shown in Fig. 1. The antenna consists of a patch, substrate 1 and 2, a rectangular OEMF, and a cavity-backed structure.

The dimensions of the patch are $W_{2 x} \times L_{2 y}$ and it is placed on the substrate 2 at height $h_{2}$. The antenna is fed through

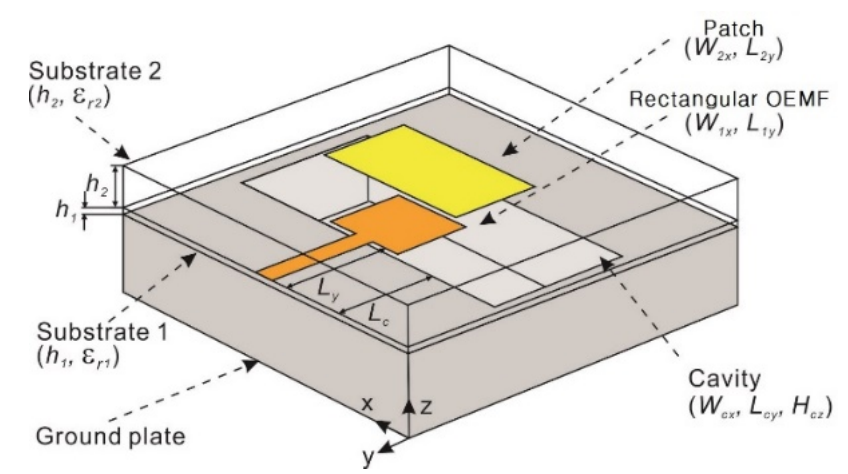

(a)

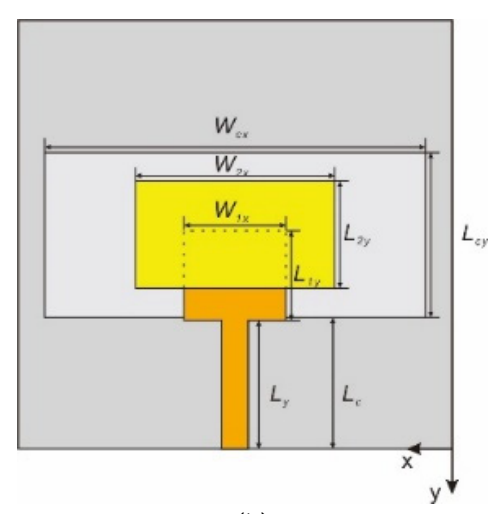

(b)

Fig. 1. Antenna configuration. (a) 3D shape and (b) top view. proximity coupling between the patch and the rectangular OEMF. The rectangular OEMF has a size of $W_{1 x} \times L_{1 y}$, and the length of the feed line is $L_{y}$. The length between the cavity starting point and ground end on the $y$ axis is $L_{c}$. A ground plane with a cavity is placed below substrate 1 . The antenna parameters were $\varepsilon_{r 1}=\varepsilon_{r 2}=2.2, W_{2 x}=13.72 \mathrm{~mm}, L_{2 y}=7.25$ $\mathrm{mm}, h_{1}=0.508 \mathrm{~mm}, h_{2}=3.175 \mathrm{~mm}, W_{1 x}=7 \mathrm{~mm}, L_{1 y}=6.25$ $\mathrm{mm}, L_{y}=9 \mathrm{~mm}, L_{c}=9 \mathrm{~mm}, W_{c x}=11.65 \mathrm{~mm}, L_{c y}=26.58 \mathrm{~mm}$, and $H_{c z}=3.74 \mathrm{~mm}$.

Variations of the design parameters of the cavity, patch and rectangular OEMF affect the input impedance and resonant frequency of the antenna. In the following parameter study, only a rectangular OEMF and the parameter of focus are varied, and the remaining parameters are maintained as presented above.

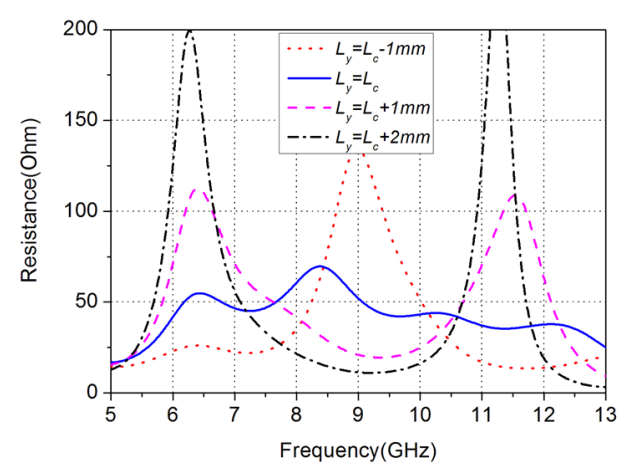

(a)

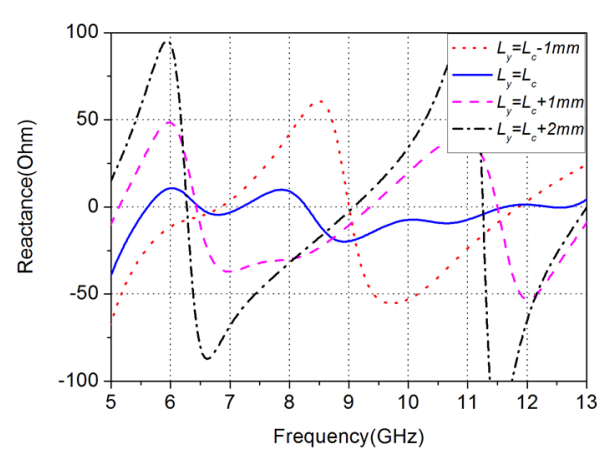

(b)

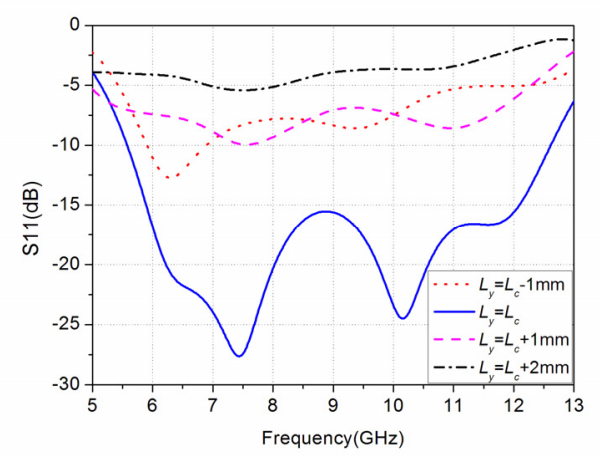

(c)

Fig. 2. Input impedance and reflection coefficient of the antenna with variation of length of the rectangular $\operatorname{OEMF}\left(L_{y}\right):$ (a) resistance, (b) reactance, and (c) reflection coefficient. 
Fig. 2 shows the variation of input impedance and reflection coefficient when the length of the rectangular $\operatorname{OEMF}\left(L_{y}\right)$ is changed. In case of $L_{y}>L_{c}$, upper and lower resonances occur, but the impedance between the two resonances is not matched to $50 \Omega$. In the case of $L_{y}=L_{c}$, the impedance is roughly $50 \Omega$ and the impedance variation is small, which means that broadband matching occurs. In the case of $L_{y}<L_{c}$, a single resonance occurs near $9 \mathrm{GHz}$, and the resistance in the other frequencies is smaller than $50 \Omega$ and the variation of the reactance increases. Consequently, the broadband matching is broken. $S_{11}$ also has the largest bandwidth when $L_{y}=L_{c}$.

Variations of input impedance when all parameters are the same and only $L_{1 y}$ is changed, meaning the length of the $y$ -

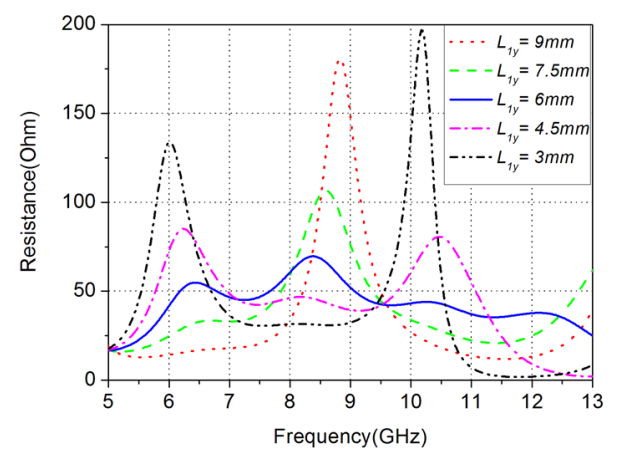

(a)

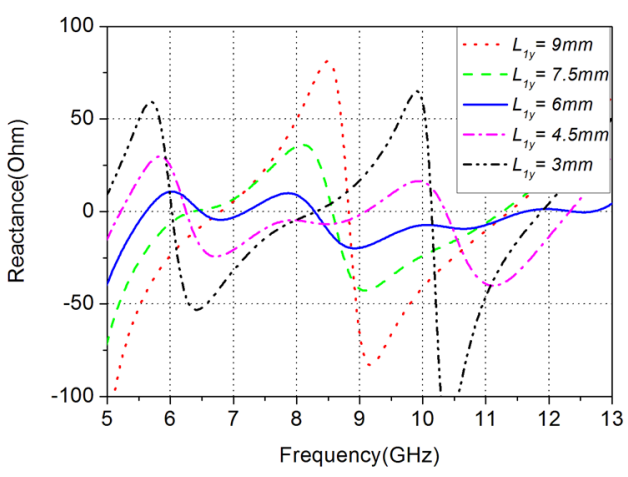

(b)

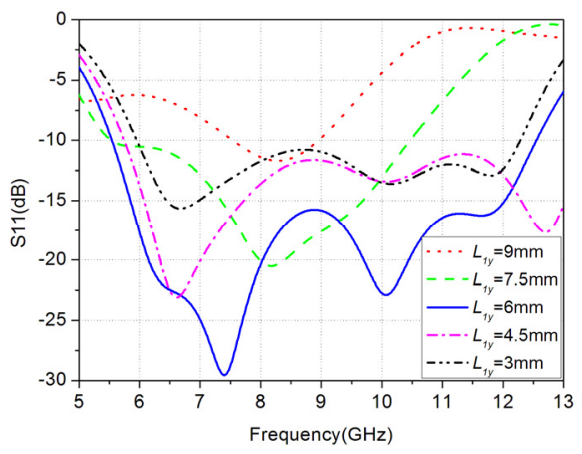

(c)

Fig. 3. Input impedance and reflection coefficient of the antenna with variation of size of the rectangular $\operatorname{OEMF}\left(L_{1 y}\right)$ : (a) resistance, (b) reactance, and (c) reflection coefficient. direction of the rectangular OEMF when the end of the rectangular OEMF is aligned with the center of the top patch, are shown in Fig. 3. It can be seen that when $L_{1 y}$ is $6 \mathrm{~mm}, S_{11}$ has the widest bandwidth characteristic. When $L_{1 y}$ is larger than 6 $\mathrm{mm}$, a single resonance occurs. As $L_{1 y}$ becomes smaller, the resonance peak decreases. It can be seen that broadband matching occurs when the end of the cavity and the starting point of the rectangular open ended microstrip coincide on the $y$-axis $\left(L_{1 y}=\right.$ $6 \mathrm{~mm})$.

Fig. 4 shows the change in reflection coefficients when the dimension of the cavity is varied. As $L_{c y}$ became larger, broadband matching occurred. $W_{c x}$ showed a bandwidth increase when it was increased to $12 \mathrm{~mm}$. $H_{c z}$ exhibited good bandwidth at about $4 \mathrm{~mm}$ and when $H_{c z}$ was too low, bandwidth was sig-

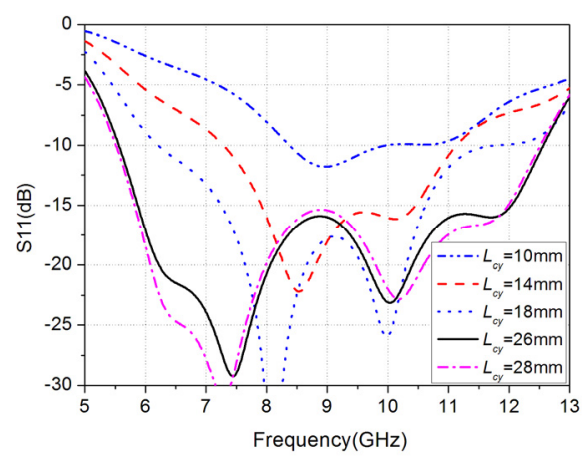

(a)

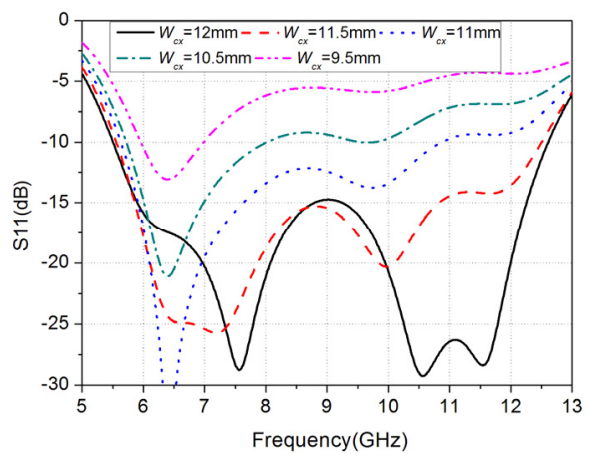

(b)

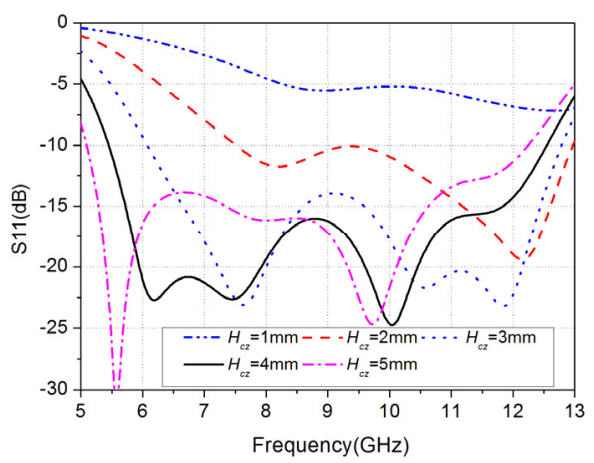

(c)

Fig. 4. Reflection coefficients of the antenna with different sizes of cavity: (a) $L_{c y}$, (b) $W_{c x}$, and (c) $H_{c z}$. 


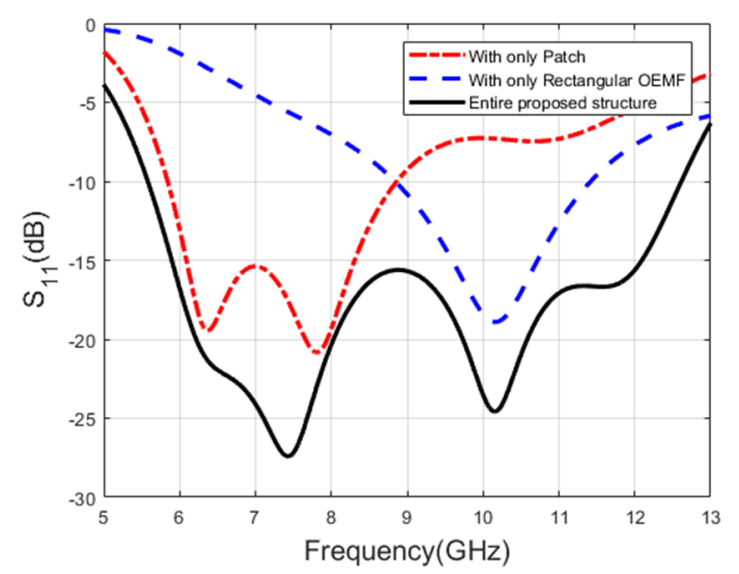

Fig. 5. Simulated reflection coefficient of the antenna with different structures.

nificantly reduced. It can be seen in Fig. 4 that additional resonance in the upper frequency band occurs, especially when the cavity is large $\left(L_{c y} \geq 26 \mathrm{~mm}, W_{c x} \geq 11.5 \mathrm{~mm}, H_{c z} \geq 3 \mathrm{~mm}\right)$.

Fig. 5 shows results of $S_{11}$ for the structure with only the patch, only the rectangular OEMF, and the whole proposed structure. The antenna with only the patch is for the structure where only the narrow feed line is extended with the same length. Only the rectangular OEMF is for the structure without the patch and the substrate 2 . The $S_{11}$ characteristic of the structure with only the patch $\left(W_{1 x}=1.8 \mathrm{~mm}\right)$ has a bandwidth of approximately $26 \%$ in the low band of 6 to $9 \mathrm{GHz}$, which shows the bandwidth performance when the cavity is coupled to the proximity-coupled antenna. The $S_{11}$ characteristic of the structure with only the rectangular OEMF $\left(W_{2 x}=0 \mathrm{~mm}\right)$ has a bandwidth of approximately 20\% in the high band of 9 to 12 $\mathrm{GHz}$, which shows the bandwidth expansion effect of the rectangular OEMF on the entire structure. The rectangular OE$\mathrm{MF}$ allows additional bandwidth to be secured while maintaining the existing bandwidth. The $S_{11}$ characteristics of the proposed structure are shown by combining the characteristics of the structure with only the patch and the structure with only the rectangular OEMF. Because the proximity-coupled microstrip antenna with cavity-acked structure mainly operates at the lower band and the added rectangular OEMF as a modified feed line mainly operates at the upper band, the combination of the two structures eventually increases the bandwidth.

It can be seen that a bandwidth of approximately $77 \%$ is realized from 5.6 to $12.6 \mathrm{GHz}$. It can be observed that VSWR bandwidth is almost doubled compared with the conventional method [12]. All the simulations were performed using the commercial software Computer Simulation Technology (CST) Microwave Studio.

Electric field distributions on the $y-z$ plane show the status of the resonance of patch and rectangular OEMF depending on the frequency variation. The simulated field distributions for the
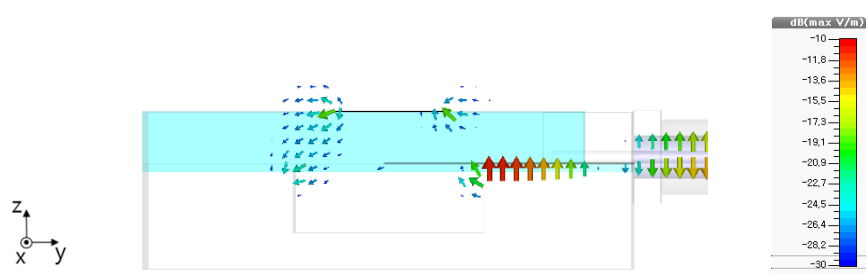

(a)
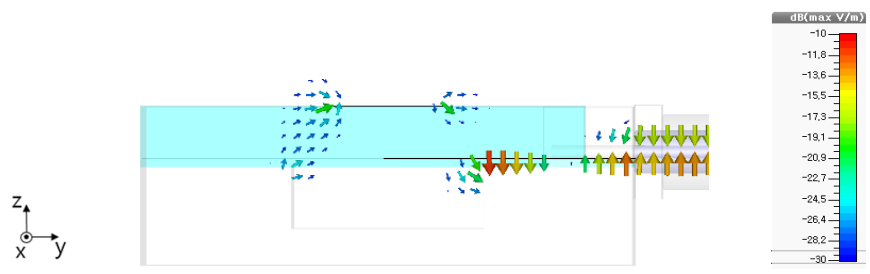

(b)
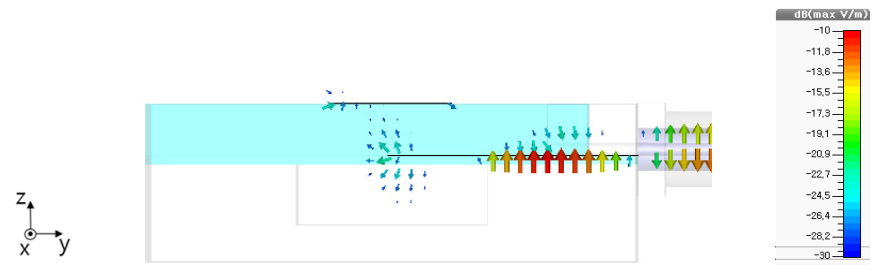

(c)
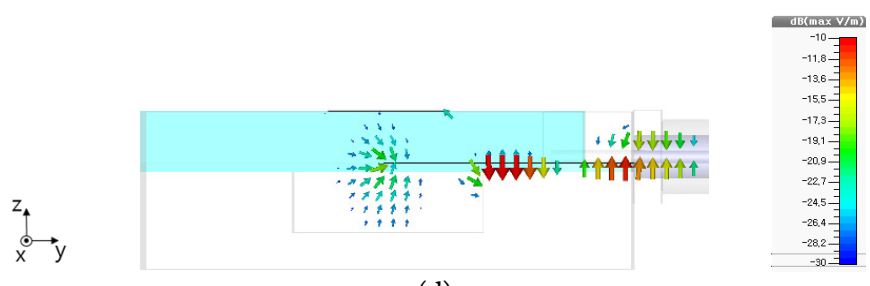

(d)

Fig. 6. Electric field distribution in the $y-z$ plane depending on frequency: (a) $6.4 \mathrm{GHz}$, (b) $7.4 \mathrm{GHz}$, (c) $10.1 \mathrm{GHz}$, and (d) $11.8 \mathrm{GHz}$.

three frequencies in the side view are shown in Fig. 6. In the case of $6.4 \mathrm{GHz}$ and $7.4 \mathrm{GHz}$, it can be seen that resonance mainly occurs in the patch. In the case of $10.1 \mathrm{GHz}$ and 11.8 $\mathrm{GHz}$, it can be seen that resonance occurs mainly in the rectangular OEMF, and very weak resonance occurs in the patch.

\section{FABRICATION AND MEASUREMENT RESULTS}

An antenna prototype was fabricated as shown in Fig. 7. To connect a coaxial connector, additional housing with aluminum was added to the side of the antenna, which only negligibly affects the antenna performance.

The simulation and measurement results of the co-polarization patterns at $6,8,10$, and $12 \mathrm{GHz}$ for $\mathrm{E}$-plane and $\mathrm{H}$ plane are presented in Fig. 8. The measured results were found to be consistent with the simulated ones. The asymmetry of the E-plane radiation pattern gradually tends to increase with the increase in frequency.

At $12 \mathrm{GHz}$, a null is generated in approximately $60^{\circ}$ direction, 


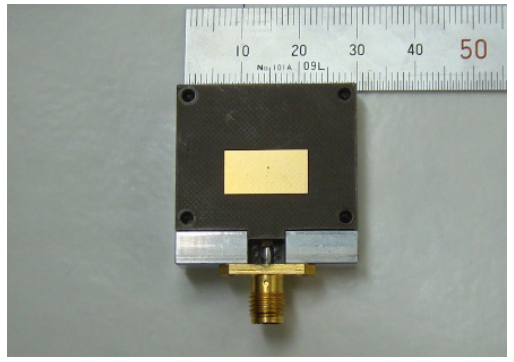

(a)

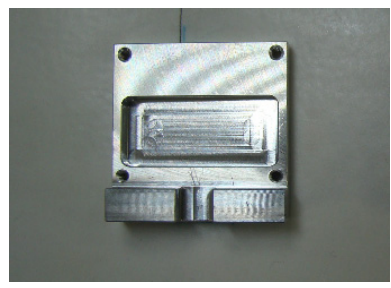

(b)

Fig. 7. Prototype of the proposed antenna (a) and cavity structure (b).

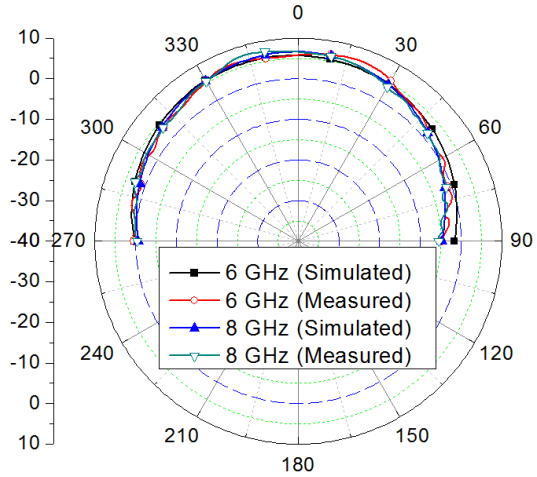

(a)

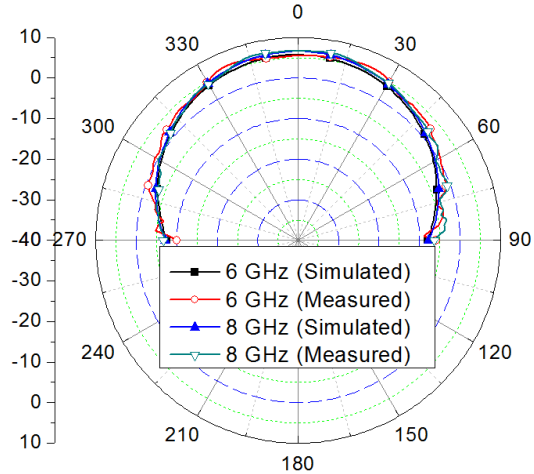

(c)

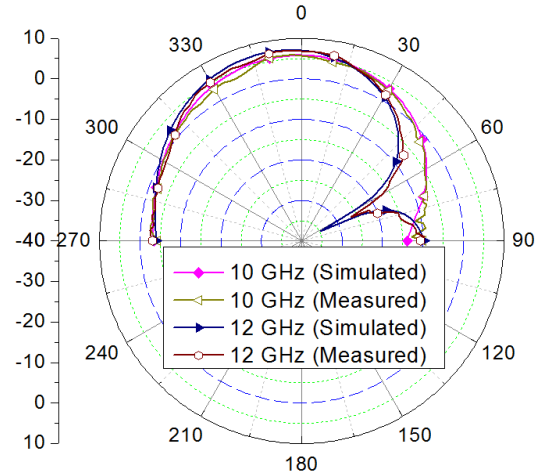

(b)

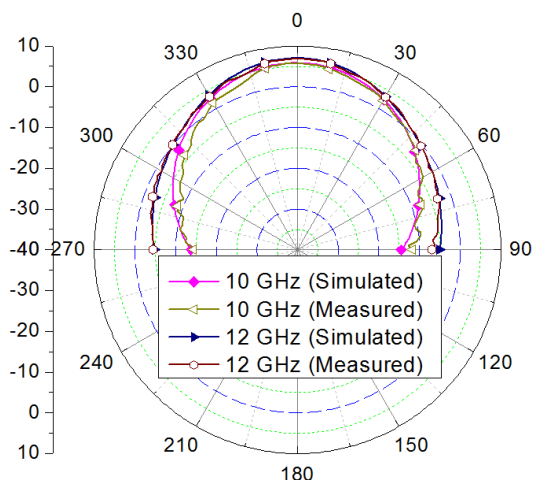

(d)

Fig. 8. Measured and simulated radiation patterns of the antenna at different frequencies: (a) E-plane (6, $8 \mathrm{GHz}),(\mathrm{b}) \mathrm{E}$-plane (10, 12 $\mathrm{GHz}),(\mathrm{c}) \mathrm{H}$-plane (6, $8 \mathrm{GHz})$, and (d) H-plane (10, $12 \mathrm{GHz})$.

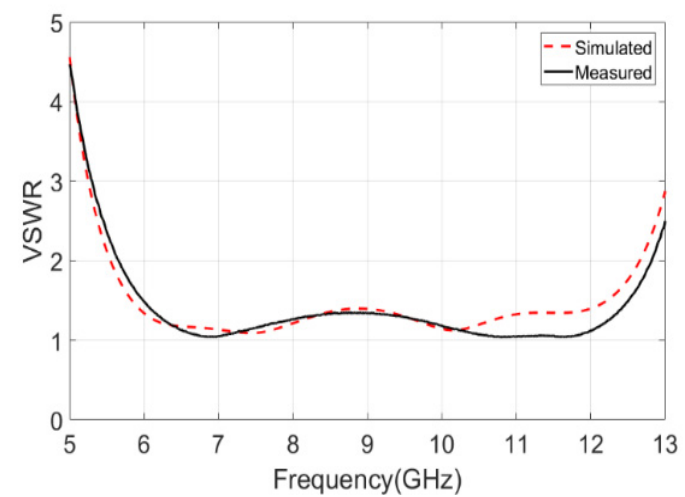

Fig. 9. Simulated and measured VSWRs for the proposed antenna.

which causes a narrowing of the antenna beam width. The radiation pattern was found to be distorted due to the physical

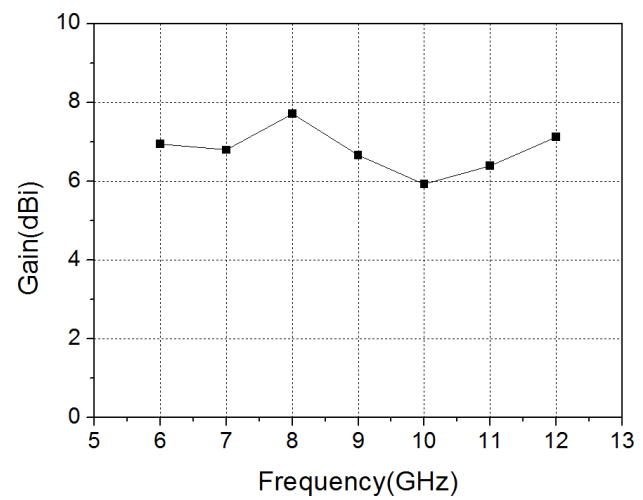

Fig. 10. Measured gain of the proposed antenna.

asymmetry of the antenna on the $z-y$ plane.

As shown in Fig. 9, the VSWR was simulated and measured. 
The VSWR bandwidth was measured to be more than $77 \%$ for the proposed antenna. The measured result was consistent with the simulated one.

Fig. 10 shows the measured gains of the proposed antenna. A reasonably good radiation gain from $6 \mathrm{dBi}$ to $8 \mathrm{dBi}$ is obtained within the entire operating band.

\section{CONCLUSION}

This paper proposed a broadband modified cavity-backed proximity-coupled microstrip patch. The patch was designed to resonate with the cavity in the lower band and the rectangular OEMF to resonate with the cavity in the upper band, resulting in a bandwidth as high as $77 \%$ (VSWR $\leq 2.0$ ) across a frequency range of 5.6 to $12.6 \mathrm{GHz}$. The proposed antenna configuration with a cavity-backed ground plane is easy to fabricate and is expected to be applicable to wideband array antennas.

\section{REFERENCES}

[1] W. S. Rowe and R. B. Waterhouse, "Investigation into the performance of proximity coupled stacked patches," IEEE Transactions on Antennas and Propagation, vol. 54, no. 6, pp. 1693-1698, 2006.

[2] Y. X. Guo, C. L. Mak, K. M. Luk, and K. F. Lee, "Analysis and design of L-probe proximity fed-patch antennas," IEEE Transactions on Antennas and Propagation, vol. 49, no. 2, pp. 145-149, 2001.

[3] S. W. Qu and Q.Xue, "A Y-shaped stub proximity coupled V-slot microstrip patch antenna," IEEE Antennas and Wireless Propagation Letters, vol. 6, pp. 40-42, 2007.

[4] B. L. Ooi, "A double- $\pi$ stub proximity feed U-slot patch antenna," IEEE Transactions on Antennas and Propagation, vol. 52, no. 9, pp. 2491-2496, 2004.

\section{Deok Kyu Kong}

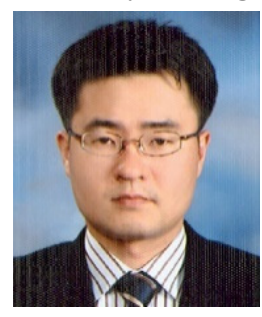

received his B.S. and M.S. degrees in electrical and computer engineering from Ajou University, Suwon, Korea in 1994 and 1996, respectively. He joined the Agency for Defense Development, Daejeon, Korea, in 1996, where he is currently a Principal Researcher. His current research interests include wideband antennas and direction-finding antennas.
[5] A. S. Elmezughi, W. S. T. Rowe, and R. B. Waterhouse, "Cavity backed hi-lo stacked patch antennas," in Proceedings of 2008 IEEE Antennas and Propagation Society International Symposium, San Diego, CA, 2008, pp. 2301-2034.

[6] X. D. Song, X. P. Lu, and W. Wei, "A single-layer wideband cavity-backed microstrip patch antenna," in Proceedings of 2015 IEEE International Symposium on Antennas and Propagation E USNC/URSI National Radio Science Meeting, Vancouver, Canada, 2015, pp. 2425-2426.

[7] Y. Wang, F. Zhu, and S. Gao, "Compact cavity-backed patch antenna with wideband bandwidth using parasitic patch," in Proceedings of 2017 6th Asia-Pacific Conference on Antennas and Propagation (APCAP), Xi'an, China, 2017, pp.1-3.

[8] J. Zhu, C. H. Chu, L. Deng, C. Zhang, Y. Yang, and S. Li, "mm-Wave high gain cavity-backed aperture-coupled patch antenna array," IEEE Access, vol. 6, pp. 44050-44058, 2018.

[9] R. L. Xia, S. W. Qu, S. Yang, and Y. Chen, "Wideband widescanning phased array with connected backed cavities and parasitic striplines," IEEE Transactions on Antennas and Propagation, vol. 66, no. 4, pp. 1767-1775, 2018.

[10] Y. Liu, S. Chen, Y. Ren, J. Cheng, and Q. H. Liu, "A broadband proximity-coupled dual-polarized microstrip antenna with L-shape backed cavity for X-band applications," AEU-International Journal of Electronics and Communications, vol. 69, no. 9, pp. 1226-1232, 2015.

[11] D. Sun and L. You, "A broadband impedance matching method for proximity-coupled microstrip antenna," IEEE Transactions on Antennas and Propagation, vol. 58, no. 4, pp. 1392-1397, 2010.

[12] D. Sun, W. Dou, L. You, X. Yan, and R. Shen, "A broadband proximity-coupled stacked microstrip antenna with cavity-backed configuration," IEEE Antennas and Wireless Propagation Letters, vol.10, pp. 1055-1058, 2011.

Jaesik Kim

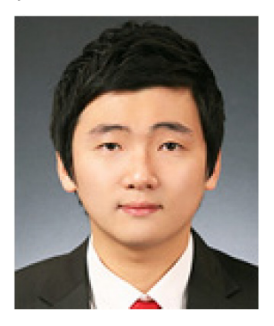

received B.S. degree in radio wave engineering from Kwangwoon University Seoul, Korea in 2011 and a $\mathrm{Ph} . \mathrm{D}$. degree in electrical and electronic engineering from Yonsei University, Seoul, Korea in 2017. He joined the Agency for Defense Development, Daejeon, Korea in 2017, where he is currently a Senior Researcher. His current research interests include direction-finding antennas and phased array antennas. 


\section{Daewoong Woo}

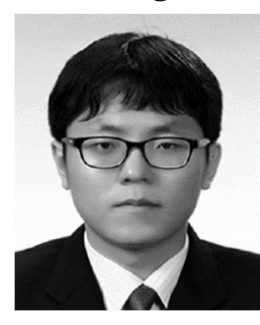

received B.S. degree in electronic and electrical engineering from Kyungpook National University, Daegu in 2007, and a Ph.D. degree in electrical engineering from Pohang University of Science and Technology (POSTECH), Pohang, Korea, in 2013. He joined the Agency for Defense Development, Daejeon, Korea, in 2013, where he is currently a Senior Researcher. His current research interests include phased array antennas, slot array antennas, periodic structures and direction-finding.

\section{Young Joong Yoon}

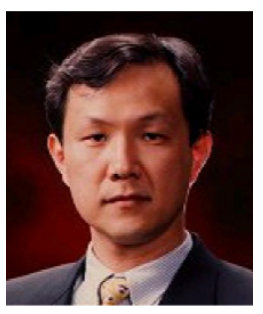

received B.S. and M.S. degrees in electronic engineering from Yonsei University, Seoul, Korea, in 1981 and 1986, and a Ph.D. in electrical engineering from the Georgia Institute of Technology, Atlanta, in 1991. In 1993, he joined the Department of Electrical and Electronic Engineering, Yonsei University, where he is currently a Professor of Electromagnetics. His main research interests are related to microwave theory, small antennas, high-power electromagnetics, and radio propagation. 